\title{
Autonomous Information Fading and Provision to Achieve High Response Time in Distributed Information Systems
}

\author{
Xiaodong $\mathrm{Lu}^{*}$ Non-member \\ Helene Arfaoui* Non-member \\ Kinji Mori* Member
}

\begin{abstract}
In highly dynamic electronic commerce environment, the need for adaptability and rapid response time to information service systems has become increasingly important. In order to cope with the continuously changing conditions of service provision and utilization, Faded Information Field (FIF) has been proposed. FIF is a distributed information service system architecture, sustained by push/pull mobile agents to bring high-assurance of services through a recursive demand-oriented provision of the most popular information closer to the users to make a tradeoff between the cost of information service allocation and access. In this paper, based on the analysis of the relationship that exists among the users distribution, information provision and access time, we propose the technology for FIF design to resolve the competing requirements of users and providers to improve users' access time. In addition, to achieve dynamic load balancing with changing users preference, the autonomous information reallocation technology is proposed. We proved the effectiveness of the proposed technology through the simulation and comparison with the conventional system.
\end{abstract}

Keywords: faded information field (FIF), information service system, push/pull mobile agent (Push/Pull-MA), load balancing

\section{Introduction}

The advent of electronic commerce has revolutionized the way that contemporary business operates. Nowadays, online purchasing in becoming widely adopted and its selling volume is continuously increasing. Not only does electronic commerce reduces the operation cost of a business, but also makes a service available to customers in anytime and from anywhere. As a result the needs are becoming more stringent and complex to cope with rapidly changing user demands, network status and information contents. Continuously, new services are added, others are updated, destroyed or in fault, making it more and more difficult to maintain. Obviously, the complexity and the business criticalness of those systems foster an urgent need for high-assurance in those systems ${ }^{(1)}$.

Service utilization varies in quantity and quality, and consequently the complete service offer is generally irrelevant to users. Analyzing the server logs of some HTTP servers, a study has shown that more than $80 \%$ of the generated traffic was generally caused by accesses to less than $6 \%$ of the actual available information on the central server ${ }^{(2)}$. In order to reduce network traffic and improve users' access time, we are developing a demand-oriented architecture called Faded Information Field (FIF) sustained by push/pull mobile agents ${ }^{(3)}$. In the FIF, the service provider allocates its popular in-

\footnotetext{
* Department of Computer Science, Tokyo Institute of Technology

2-12-1 Ookayama, Meguro, Tokyo 152-8552
}

formation to adjacent nodes, close to the users. The characteristic of the FIF is to balance the cost of information allocation performed by push mobile agents and the cost of the access to the information performed by pull mobile agents ${ }^{(4)}$. The information structure consequently permits to preserve the same access time to all unspecified users, whatever their current demand trend and volume.

In this paper, the relationship that exists among the users distribution, information fading volume and the access time of pull mobile agents is clarified. Based on these factors, we propose the technology for FIF design to resolve the competing requirements of users and providers to improve response time. However, when users' preference changes, the system that originally was designed to have balanced load may lose this balance. In order to achieve dynamic load balancing in rapidly changing environments, autonomous information reallocation technology is proposed just based on the current situation of the locality.

The structure of the paper is organized as follows. In the next section, the application and system requirements are described. In section 3, the concept of autonomous information service system and the architecture of FIF is presented. Problem formulation and the technology to solve it for FIF construction are presented in section 4 . Section 5 presents the autonomous information reallocation technology for dynamic load balancing. The simulation in section 6 shows that the proposed technology improves response time to compare with conventional models. The last section is the conclusion of 
the paper.

\section{Requirements}

The traditional mode of information services provision has been focused on the relatively static environments of centralized management systems, with most of the processing, storage and retrieval of data performed at central sites. Consequently, both communication with and processing at the central site have become system bottlenecks, response speeds have slowed down, and the availability and reliability of information services has deteriorated.

Under dynamically changing situation, information service systems are characterized by continuous changes of services from service providers promoting their offers and changing users' demand. In such system, service providers require to provide non-stop services with timely update, and at the same time, users require timely access to information services corresponding to their individual needs ${ }^{(5)}$.

Therefore, there is a need for a system able to consider both users' requirements for rapidly accessing the services and SPs' requirements for providing just in time new services. The system needs for demand-oriented access to services ${ }^{(6)}$, which can cope with the application needs mentioned above are summarized as adaptability and timeliness.

\section{Autonomous Information Service System}

3.1 Concept The main concern of the information service system in the past has been to efficiently retrieve from enormous repositories the relevant information for a particular request. In order to provide high-assurance of information services in dynamically changing environment, we have developed a system architecture supported by mobile agent infrastructure that stores pushed information services and executes pulled users requests. Based on the concept of Autonomous Decentralized System (ADS) ${ }^{(7)}$, the new concepts for information provision and access of Autonomous Information Service System are proposed.

- Autonomous Information Provision: Each service provider pushes its accessibility towards users to promote its contents.

- Autonomous Information Utilization: Each user pulls requested data corresponding to his requirements.

3.2 The Architecture of Faded Information Field The main goal of the faded information field is to guarantee the assurance of autonomous information service provision and utilization. Service providers trace the demand trend for information, and allocate to accepting storing nodes the most accessed segment of their information services. The storing nodes then, in a recursive pruning process, further allocate the information services to adjacent nodes. As a result, the multi-level distributed information services area is created. Nodes adjacent to the SP contain more information but are also far from users, while outmost nodes

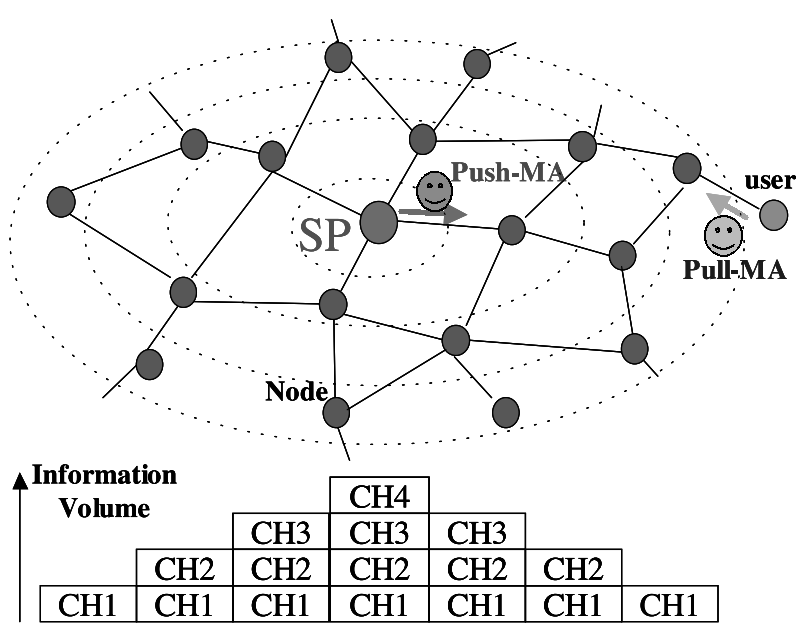

Fig. 1. FIF Architecture

storing the minimum information amount, are located at the edge of the users' network. Users with different requirements for information can be satisfied at different levels in the FIF. Consequently, the cost of service utilization (access time) and cost of service provision (update) are balanced by allocating closer to the majority of the users the most accessed part of the information services. Fig. 1 shows the FIF architecture and the information volume on each level. Content Code (CC) and Characteristic Codes (CH1...CH4) will be explained in subsection 3.4.

The FIF architecture is based on the demand-oriented replication of the information services to warrant services availability and responsiveness. In the transaction processing system that data consistency of all replicas at all times must be assured, and the updates are propagated synchronously. However, FIF is not a transactionoriented system. The replica management protocol in the FIF just provides weaker consistency guarantees, and the updates performed by push mobile agents are asynchronous in a certain period.

The process of information provision by SPs as described above, is called push technology. The process of searching information is called pull technology ${ }^{(8)}$. We propose Push Mobile Agents (Push-MAs) for distributing information and Pull Mobile Agents (Pull-MAs) for searching information on the network as shown in Fig. 1. In the next subsection, we shall describe mobile agents and their role in the proposed system.

3.3 Autonomous Subsystems Mobile agent technologies ${ }^{(9)}{ }^{(10)}$ are utilized in FIF as the medium for a dynamic interaction between information service provision and utilization. In the FIF model, each action is given to a mobile agent that bridges the autonomous entities. The FIF is composed of the following three autonomous subsystems.

(1) Push-MA: The SP generates Push-MAs, and delegates them the task of information fading. It is responsible for allocating information based on the SP's requirements for timeliness and reliability. Push-MAs autonomously coordinate with nodes for fading, and selecting appropriate infor- 


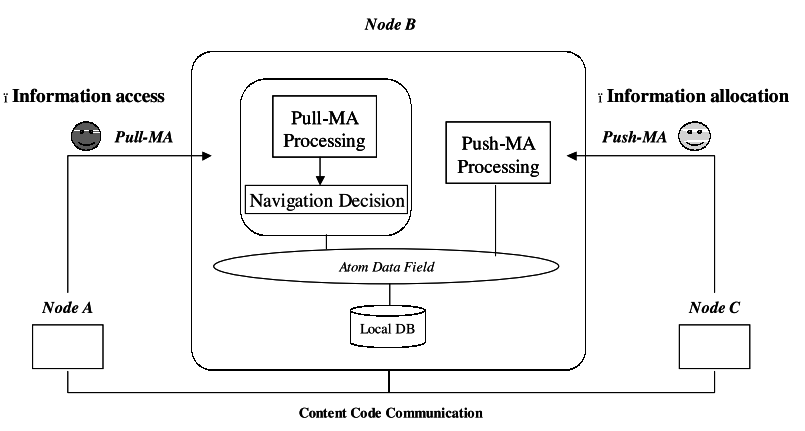

Fig. 2. Node agent platform

mation according to the requirement of the node and importance level of the information.

(2) Pull-MA: A user who requires information transmits a customized Pull-MA into the network. Pull-MA autonomously navigates through the distributed nodes in FIF to find out step-by-step more relevant information.

(3) Node: Fig. 2 shows the agent-execution platform of each node to process push/pull mobile agents. It is responsible for monitoring the system conditions based on local information and accepting to receive allocation request. It autonomously coordinates with Push-MAs to determine upper directions to the information services, and with Pull-MAs to determine the response time on each of its upper directions.

Every subsystem has autonomous controllability over its own operations and autonomous controllability with the other subsystems to continue its operations even under evolving situations ${ }^{(11)}$. Information provision and access in the FIF is based on content code communication to be described in the next subsection.

3.4 Content Code Communication Conventional communication methods use the destination address to send the data. In highly changing environment, the state of the nodes, the stability of the connections, the status of the SPs are highly unpredictable and maintaining repository of the SP addresses cannot guarantee high-assurance of the system ${ }^{(12)}$. In the FIF information contents are uniquely defined by Contents Code (CC). The information contents are further specified by its Characteristic Codes $(\mathrm{CHs})$. For instance, the CC identified by CC1 standing for PC Store can be further specified by the CHs: Product ID, Price, CPU, Memory and Options as shown in Fig. 3. Service providers transmit information services through Push-MAs by specifying content codes (CCs) of information to the nodes using message format shown in Fig. 3. The nodes receive information and select to store autonomously based on CCs. Users search information based on CCs by exploiting Pull-MAs.

3.5 Autonomous Fading Autonomy of SP and nodes is the main aspect of FIF construction. Nodes may select to store a portion of the information depending upon their situation. Service providers need to send certain parameters to the nodes to indicate the priority of $\mathrm{CHs}$ and specify the amount of information

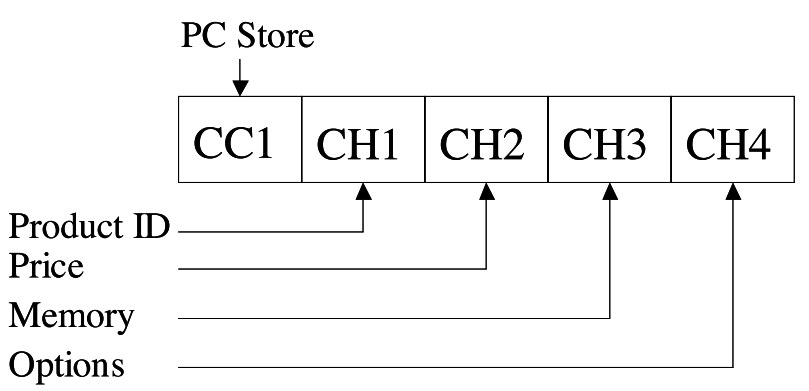

Fig. 3. Communication message in the FIF

to be faded. We consider information represented by $\mathrm{CC}$ at the $\mathrm{SP}$ with $\mathrm{CH} 1$ to $\mathrm{CH} 4$. We assume that $\mathrm{CH} 4$ is the least popular and $\mathrm{CH} 1$ is the most popular. The information transmission starts from SP to adjacent nodes through Push-MAs. The $\mathrm{CH}$ indicates amount of information and importance of information. The autonomous fading process can be described in following steps:

(1) After receiving the Push-MA, the node checks all fields of message, and stores the amount of information depending on its situation and the priority of CHs.

(2) Push-MA fades the remained CHs not selected by the node.

(3) The node recorders the neighbor from which it receives the Push-MA as a upper node.

(4) And then the node sends the Push-MA to other adjacent nodes.

Also, a node in the system may receive Push-MAs from many nodes. In this case, the node checks all fields to identify the source, version and amount of information and stores only necessary portion of information. As a result, the most accessed $\mathrm{CH}$ is stored on all nodes and the multi-level faded information field is created, as shown in Fig. 1.

3.6 Autonomous Navigation The goal of autonomous navigation is to offer load balancing, to assure the dynamic dispatching of the Pull-MAs. A node in the system receives directly Pull-MAs from the lower layers and only has a view of its upper nodes. The path congestion, which is defined as the mean of the access times of the returning Pull-MAs, is an expression of the average processing time for that path. Each node monitors the path congestion and analyzes the time differences on each path. The autonomous dispatching must assure that all path processing time be equal. If the current average processing time of a path is different with other paths values, the departure rate to the upper paths is then accordingly modified. The complete mechanism of the autonomous navigation is discussed in ${ }^{(13)}$.

\section{FIF Construction to Achieve High Response Time}

The size of FIF and amount of information volume at each level is an important consideration for design of FIF. In the FIF, the total access time for the users consists of Push-MA's distribution time and Pull-MA's access time. The objective of this section is to clarify the 
Push-MA's information distribution time and Pull-MA's access time to determine the total users' information access time.

4.1 Information Provision Time SP generates Push-MAs to allocate information in the network based on the importance of the information and the capacity of the node. Push-MA traverses from the upper node to lower node, and stores certain information, while fading others in coordination with the node, and moves to the next node. Fig. 4 shows two fading models of the FIF which the decrease of the information volume is different on each level. If $I(j)$ is the amount of information volume at distance $j$ from the SP, $I_{\text {push }}$ is the code size of Push-MA, $T_{p j}$ is the process time at level $j$ and time to transmit unit information to adjacent node is $\lambda$ then the information distribution time $T_{\text {push }}$ of Push-MA at distance $m$ from the SP can be given by:

$$
T_{p u s h}=\sum_{j=1}^{m} I(j) \lambda+T_{p j}+m I_{p u s h} \lambda \cdots \cdots \cdots \cdots
$$

From above equation we can see that Push-time depends on the information volume stored on each level and the size of FIF. And as shown in Fig. 4, if the attenuation of the information volume is large, within same push time the most accessed information can be pushed nearer to users than the small one.

4.2 Information Access Time A user generates Pull-MA to search information in the network. Fig. 5 shows the access model of a Pull-MA. First, the

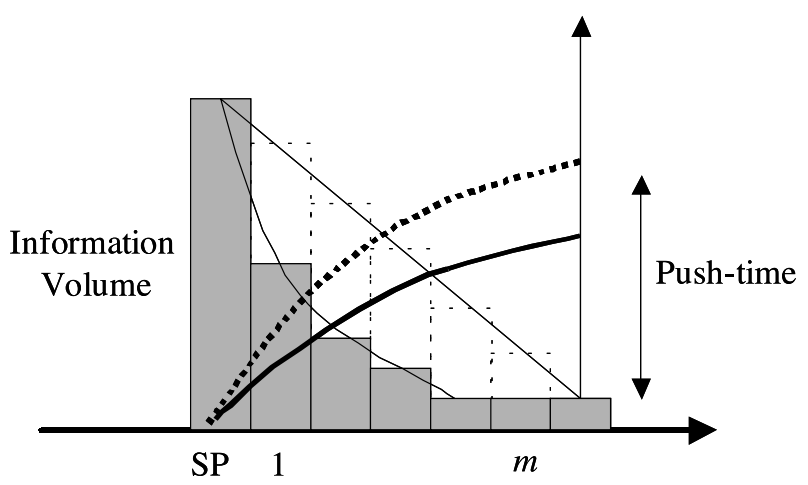

Fig. 4. Information distribution time of Push-MA

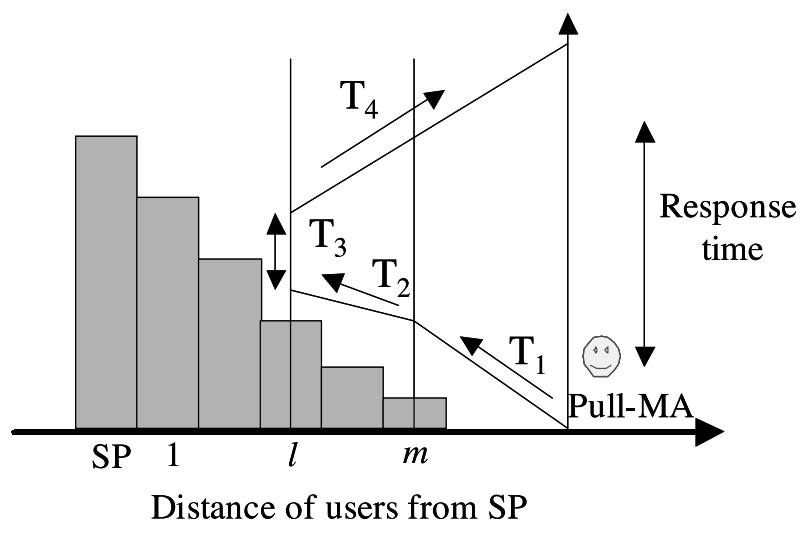

Fig. 5. Information access time of Pull-MA
Pull-MA traverses from the user's node to search the required FIF. And then in the FIF, Pull-MA traverses from lower nodes to upper nodes guided by the gradient of the information, and retrieves the required information at certain node inside the FIF. Finally, the Pull-MA carries the retrieved information to the user. The information access time $T_{\text {pull }}$ of Pull-MA consists of the time to search the FIF, time to move inside the FIF to the node which has required information volume, the time of processing at the node, and the time of return to the users as shown below:

$$
\begin{aligned}
T_{p u l l}= & S(n-m) I_{\text {pull }} \lambda+(m-l) S_{f} I_{\text {pull }} \lambda \\
& +u(I(l))+(n-l)\left(I(l)+I_{\text {pull }}\right) \lambda \ldots .
\end{aligned}
$$

Where $S(x)$ is the ratio of the time to search FIF at distance $x$ away from the FIF, $S_{f}$ is the ratio of the time for moving one node in the FIF, $u(i)$ is the time for access information amount $i$ at a node, $I(l)$ is the amount of information get at node $l$ and $I_{\text {pull }}$ is size of Pull-MA.

4.3 Trade-off The construction of FIF to improve users' access time is the goal of SP to enhance the response time. The evaluation of the proposed technique is based on the Pull-MA's access time considering all Pull-MAs in the system. The access time of the PullMA changes as the search time improves due to the expansion of the FIF, however, navigation time in the FIF may become large because the Pull-MA may proceed to higher levels. There are two major outcomes of this change. Firstly, there are some Pull-MAs, say $a$, which can be satisfied at the boundary of the extended FIF. Secondly, there are other Pull-MAs, say $b$, which cannot be satisfied at the boundary of the extended FIF, rather they traverses into the FIF. Considering the change in the size as $d$, the total decrease in access time $X_{a}$ of all the Pull-MAs is given by:

$$
\begin{aligned}
X_{a}= & \left(S\left(n-m_{1}\right)-S\left(n-m_{2}\right)\right)(a+b) I_{p u l l} \lambda \\
& +\left(I(m)+I_{p u l l}\right) d a \lambda+S_{f} d b I_{p u l l} \lambda \ldots \ldots
\end{aligned}
$$

The access time of certain Pull-MA will increase, as the information volume of the nodes inside the FIF attenuated due to the expansion. The expansion at each level costs $1 \mathrm{CH}$ and 1 Pull-MA code size, which is balanced by the attenuation of the information volume inside the FIF. The number of Pull-MAs satisfied at or near the boundary of the FIF decreases, as the information volume decreases, and congestion inside the FIF increases. Therefore, we introduce the congestion parameter $c(d)$ in the increase of access time of all the Pull-MAs and distance as its variable. If $N_{c h}$ is the number of CHs, $N(i)$ is the number of users who need $i$ information and $I_{E}$ is the information volume decreased at each step then the increase in total Pull-MAs access time $X_{b}$ is shown below:

$$
\begin{aligned}
X_{b}= & {\left[\left(\sum_{i=2}^{N_{c h}} I(i) N(i)+2 b S_{f} I_{p u l l}\right) /\left(N_{c h}-1\right)\right] } \\
& \times\left[\left(I_{E}+I_{\text {push }}\right) / I_{E}\right] c(d) d \lambda \ldots \ldots \ldots \ldots \ldots
\end{aligned}
$$

As depicted in Fig. 6, the equilibrium of the access 


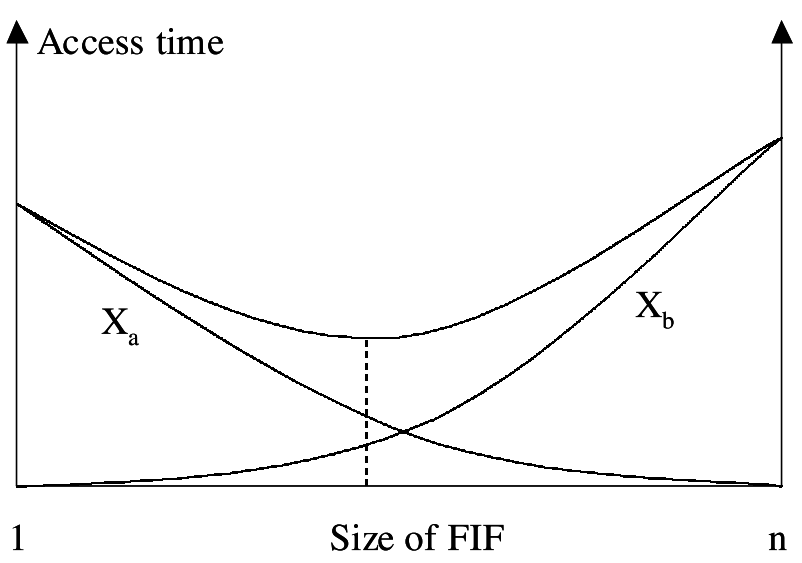

Fig. 6. The relation between $X_{a}$ and $X_{b}$

time of all Pull-MAs can be found from $X_{a}$ and $X_{b}$. As $X_{a}$ is the decrease in the Pull-MAs' access time and $X_{b}$ is the increase in the access time of the Pull-MAs, therefore the optimal point is the minimum of the sum of $X_{a}$ and $X_{b}$.

\section{Dynamic Load Balancing}

The system is constructed according to the certain users distribution and faded information volume. But all these go well only under the condition that the real users preference is roughly same as originally predicted and no changes ever since. However, as matter of common sense, users preference changes and that is unpredictable. This fosters a need for a technique to achieve dynamic load balancing through adjusting information volume on each node to maintain the almost same response time under any demand volume.

5.1 Measurement of Users Preference The proper information volume stored on each node is determined by the users preference. How to measure the changing preference of users is discussed in this subsection.

As shown in Fig. 7, at node D, between the time of two Push-MAs update, through rate, $r\left(t_{1}, D\right)$, is defined as the ratio of the number of outgoing Pull-MAs proceeding to the upper nodes $N_{u}\left(t_{1}, D\right)$ compared to the total number of incoming Pull-MAs at node D $N_{t}\left(t_{1}, D\right)$.

$$
r\left(t_{1}, D\right)=\frac{N_{u}\left(t_{1}, D\right)}{N_{t}\left(t_{1}, D\right)} \cdots \ldots \ldots \ldots \ldots \ldots \ldots
$$

The information adjusting process is regulated by the number of Pull-MAs circulating inside the system. But each node has no view of the overall structure, we utilize the notion of locality congestion. The locality of a node $\mathrm{N}$ is defined as the set of all upper nodes having link with the node $\mathrm{N}$, and including node $\mathrm{N}$ itself, as shown in Fig. 7.

The Pull-MAs load in the system is therefore perceived per locality of a node. Each node measures the degree of the congestion based on the load of its locality in coordination with Push-MAs (which bring the load information from the upper nodes to lower nodes), and can asynchronously contribute to balance the load in the system by regulating number of Pull-MAs toward

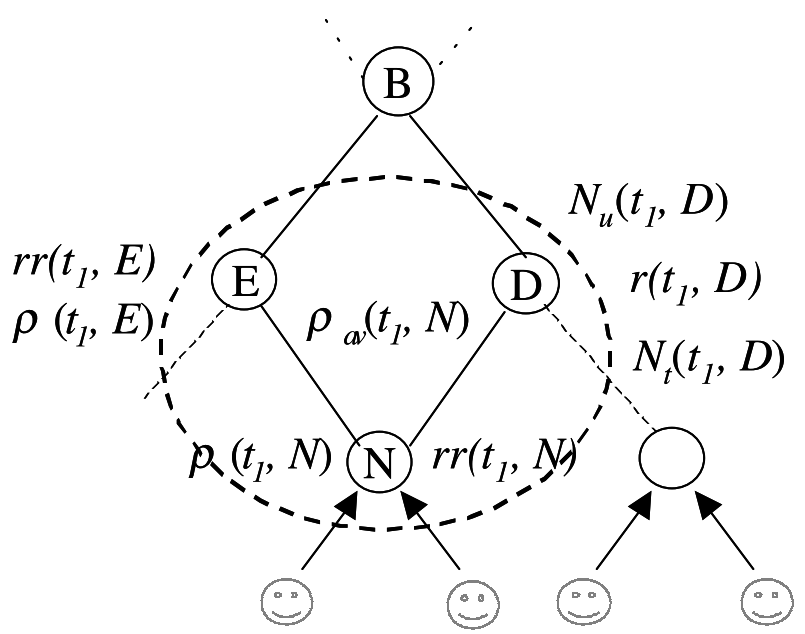

Fig. 7. Measurement of Users Preference

upper nodes. Through rate i.e. relative number of PullMAs that must proceed to upper nodes in the locality of a node $\mathrm{N}$ to achieve load balancing is called reference through rate $\operatorname{rr}\left(t_{1}, N\right)$. A node can determine reference through rate based on the congestion in the locality. The degree of congestion of a node $\mathrm{N}$ depends upon node utilization $\rho\left(t_{1}, N\right)$, the proportion of the length of the waiting queue to the capacity of the node. Each node agent measures utilization $\rho\left(t_{1}, N\right)$, and sends to lower nodes through Push-MAs. The receiving node $\mathrm{N}$ determines the average utilization $\rho_{a v}\left(t_{1}, N\right)$ of the nodes in the locality. Node $\mathrm{N}$ determines reference through rate as follows:

$$
r r\left(t_{1}, N\right)=\frac{\operatorname{rr}\left(t_{0}, N\right) \rho_{a v}\left(t_{1}, N\right)}{\rho\left(t_{1}, N\right)} .
$$

Consequently reference through rate gives a relative measure of the number of Pull-MAs that are required to proceed to upper nodes to equalize the load on each node in the locality. Therefore, a node is required to allocate information in proper amount to satisfy the reference through rate.

5.2 Autonomous Reallocation Based on the FIF construction technology, SP sends Push-MAs to allocate the most accessed segments $(\mathrm{CHs})$ of information service to nodes on the network. After receiving a Push-MA, the node stores a certain amount of popular information and fades remains from the Push-MA and sends it to the lower nodes (see Fig. 1). Then the node agent monitors the through rate and compares it with reference through rate to adjust information volume autonomously. If users trend changes, and they need more information, then through rate becomes larger than the reference through rate of a node, the node agent increases information amount upon the arrival of pushMA to bridge the gap between two rates, and vice versa. Consequently, information amount required at time $t_{2}$ at node $N$ is proportional to the ratio of through rate to reference through rate at time $t_{1}$ of the node. A node agent determines information amount $I\left(t_{2}, N\right)$ at time $t_{2}$ at node $N$ as follows: 
Table 1. Parameters of Simulation

\begin{tabular}{l|l}
\hline Parameter & Value \\
\hline$\lambda$ & $1 / 10^{5}$ \\
\hline$S_{f}$ & 1 \\
\hline$S(x)$ & $x^{2}$ \\
\hline$c(d)$ & $4 \mathrm{~d} / 3$ \\
\hline$I_{\text {push }}, I_{\text {pull }}$ & $2 \mathrm{kBytes}$ \\
\hline
\end{tabular}

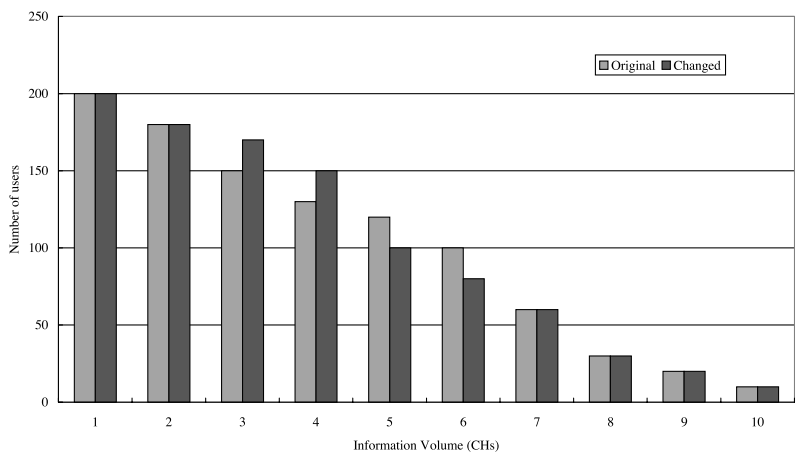

Fig. 8. The users distribution

$$
\begin{aligned}
& \text { If } r\left(t_{1}, N\right)>\operatorname{rr}\left(t_{1}, N\right) \text { then } \\
& \begin{array}{l}
I\left(t_{2}, N\right)=\left[I\left(t_{1}, N\right)+\left(I\left(t_{1}, U p N o d e\right)\right.\right. \\
\left.\left.\quad-I\left(t_{1}, N\right)\right) \frac{r\left(t_{1}, N\right)-r r\left(t_{1}, N\right)}{100-r r\left(t_{1}, N\right)}\right] \frac{I\left(t_{2}, U p N o d e\right)}{I\left(t_{1}, U p N o d e\right)}
\end{array}
\end{aligned}
$$

else

$$
I\left(t_{2}, N\right)=I\left(t_{1}, N\right) \frac{r\left(t_{2}, N\right)}{r r\left(t_{1}, N\right)} \frac{I\left(t_{2}, U p N o d e\right)}{I\left(t_{1}, U p N o d e\right)}
$$

According to this process, a node agent only needs to know the information amount and utilization of upper nodes in its locality to adjust its information volume based on the new preference.

\section{Evaluation}

In this section, we evaluate the proposed technologies by simulation. We have developed the Autonomous Information Service System Simulator (AIS3) ${ }^{(11)}$ to prove the validity of the proposed system.

6.1 Initial Settings Users have heterogeneous demands for information services, majority of the users need small portion of information. On this basis, the original distribution of users who require information volume is shown in Fig. 8 and users of changing number from 100 to 1,000 per second respectively. Total number of $\mathrm{CHs}$ is 10 and the data size of each $\mathrm{CH}$ is $1 \mathrm{kBytes}$. The distance of users from service provider is 10 hops. In Table 1 gives the initial parameter to perform simulation.

Fig. 9 shows the average access time of all Pull-MAs of different size of FIF in given users' distribution and information volume decreased at each step. To tradeoff increase and decrease of response time with changing the size of FIF, we can get that the optimal size in this case is 5 levels.

6.2 Simulation Results The first experiment is to show the effectiveness of FIF system constructed based on the proposed mechanism compared with the

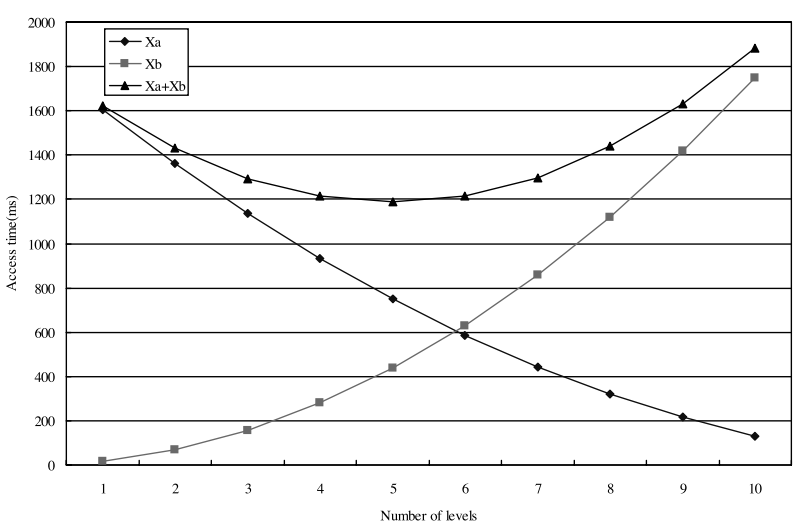

Fig. 9. Access time and the size of FIF

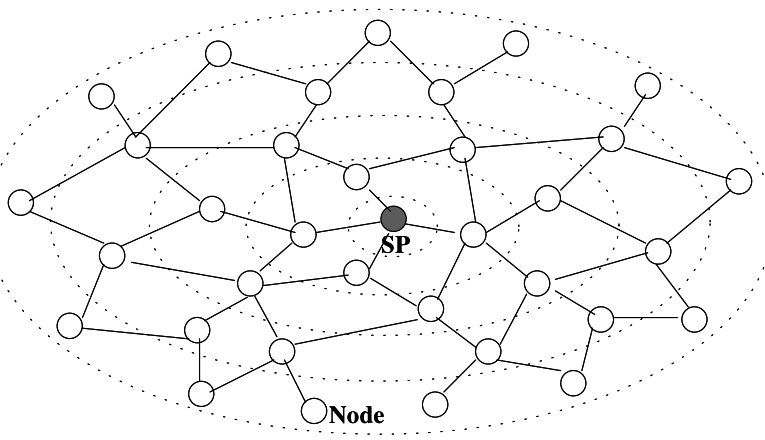

Fig. 10. The FIF model

mirror and proxy caching models. The simulation models are shown as following:

- Model1: Mirror

In this model, the nodes are distributed in the boundary layer with the whole information. But the total information volume is same as in the FIF system.

- Model2: Proxy caching

In this model, users' requests are assigned to each proxy server. For the fair comparison, the number of nodes and the total storage cost are same with that of FIF. It has been proved that a caching proxy has an upper bound of $30 \%-50 \%$ in its hit rate ${ }^{(15)}$. In the comparison the hit rate of $50 \%$ for each proxy server is applied.

- Model3: FIF

The size of FIF in this model is 5 levels. The number of nodes in each layer are 1, 4, 7, 10, and 13, respectively, as shown in Fig. 10.

As results shown in Fig. 11, FIF constructed based on the proposed technology is effective to improve the response time of users in heterogeneous requirements. Because the system is constructed taking users' preference into consideration, users' accesses are distributed into the system. So timeliness in information utilization is achieved thanks to the balanced load in the system. When accessing users are getting more, nodes in mirror and proxy caching model get much more congested. Therefore, the average response time increases but exponentially. While in the case of proposed system, because 


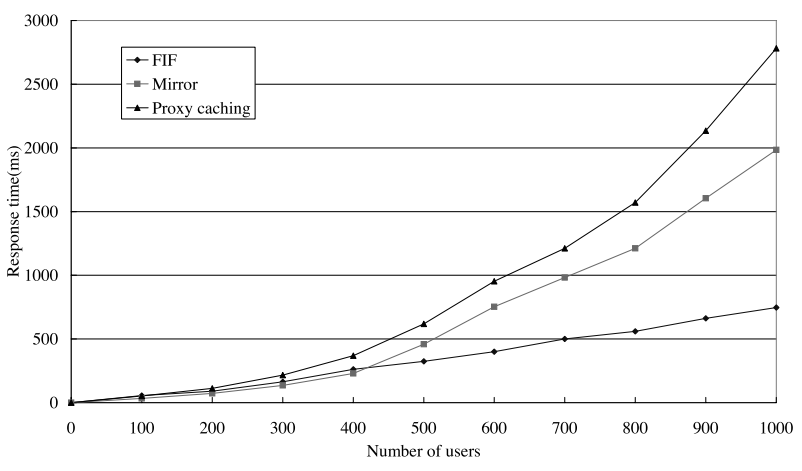

Fig. 11. Comparison of three models

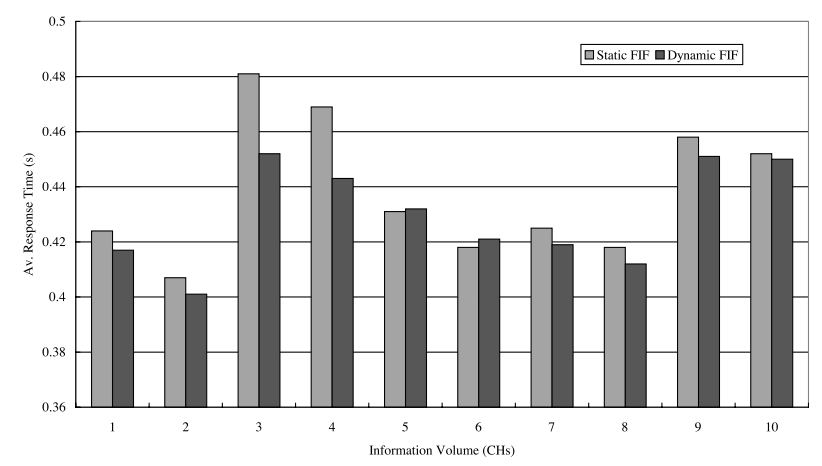

Fig. 12. Comparison of average response time

FIF leads users to the very node where it can actually be satisfied, the average response time increases but not as quickly as the situation in other two modes do. The FIF permits average $30 \%$ and $70 \%$ improvement of the response time when compared to the mirror and proxy caching models.

The second experiment mainly talks about the evaluation of autonomous information reallocation technology. To testify the effectiveness of autonomous information reallocation, the model of the change of users' preference is realized in the simulation. Here in the experiment, one basic assumption is taken about the users' preference, that the total number of users is constant. The changing of users' preference is shown in Fig. 8.

The result of comparatione between the static system, which means system without adaptation to the change, and the dynamic system applied with autonomous information reallocation is shown in Fig. 12. Referring the access model shown in Fig. 8, a rising of the number of users who require $\mathrm{CH} 3$ and $\mathrm{CH} 4$ is observed. Due to this the average response time of the static system increase. The average response time in the dynamic system keeps almost the same, which indicates that most of the users have same response time, and the system achieves load balancing.

In the FIF, users with different requirements can be satisfied at different levels. The information structure consequently permits to preserve the same access time to all unspecified users. Based on the load balancing of locality, the convergence of users access can be avoided and hence load of the system can be balanced. In addition, the network traffic is also balanced through the system architecture. So with the same refresh rate, the network traffic of FIF is obviously better than the proxy model.

Based on the discussion of results, we conclude that the FIF system architecture is highly effective to achieve not only improved users' response time but also to equalize users response time through autonomous information reallocation and achieves dynamic load balancing.

\section{Conclusion}

Recently, the necessity of achieving adaptability and timeliness of service provision and utilization has been reinforced by the introduction of new business-critical applications. It is necessary to consider both the time requirements of service provider for providing their timedependent services and the time requirements of users for accessing the information service in such dynamic systems. In that respect, FIF system architecture of pruned information replicas, has been designed to assure the timeliness and dynamic load balancing of the system in rapidly changing environment.

In this paper, we clarify the information distribution time of Push-MAs and information access time of PullMAs, and the technology for initial FIF construction based on users distribution and faded information volume is proposed. And the autonomous information reallocation technology is proposed to solve the problem that system must be updated when users' preference changed. Under evolving situation, the proposed mechanism autonomously adjust information on supplydemand basis to achieve load balancing of the incoming Pull-MAs in the locality. The evaluation results have shown the effectiveness of the proposed technology to achieve timeliness and adaptability of the system.

(Manuscript received Nov. 25, 2002, revised Sep. 6, 2004)

\section{References}

(1) I-Ling Yen, R. Paul, and K. Mori: "Toward Integrated Methods for High-Assurance Systems", IEEE Computer, Vol.31, No.4, pp.32-34 (1998)

(2) A. Bestavros: "Demand-based Document Dissemination to Reduce Traffic and Balance Load in Distributed Information System", Proc. IEEE Conf. on SPDP, pp.338-345 (1993)

( 3 ) K. Mori: "Autonomous Fading and Navigation for Information Allocation and Search under Evolving Service System", Proc. IEEE Conf. on APSITT, pp.326-330 (1999)

(4) H. F. Ahmad and K. Mori: "Autonomous Information Provision to Achieve Reliability for Users and Providers", Proc. IEEE Conf. on ISADS, pp. 65-72 (2001)

(5) X. Lu and K. Mori: "Autonomous Information Provision for High Response Time in Distributed Information System", Proc. IEEE Conf. on IWADS2002, pp. 22-27 (2002)

(6) K. Mori, S. Yamashita, H. Nakanishi, K. Hayashi, K. Ohmachi, and Y. Hori: "Service Accelerator (SEA) System for Supplying Demand Oriented Information Services", Proc. IEEE Conf. on ISADS, pp. 129-136 (1997)

(7) K. Mori: "Autonomous Decentralized Systems: Concepts, Data Field Architecture and Future Trends", Proc. IEEE Conf. on ISADS, pp. 28-34 (1993)

(8) X. Lu and K. Mori: "Autonomous Information Integration and Allocation in Agent-Based Information Service System", Proc. IEEE Conf. on IAT2003, pp. 290-296 (2003) 
(9) V. Pham, and A. Karmouch: "Mobile Sofrware Agents: An overview", IEEE Communication Magazine, Vol.31, pp. 2636 (1998)

(10) J. E. White: Mobile Agents, AAAI Press (1986)

(11) X. Lu and K. Mori: "Autonomous Integration and Optimal Allocation of Heterogeneous Information Services for HighAssurance in Distributed Information Service System", IEICE Trans. Inf. \& Syst., Vol.E86-D, No.10, pp. 2087-2094, Oct. (2003)

(12) H. F. Ahmad and K. Mori: "Autonomous Information Service System: Basic Concepts for Evaluation", IEICE Trans. Fundamentals, Vol.E83-A, No.11, pp. 2228-2235 (2000)

(13) H. Arfaoui and K. Mori: "Autonomous Navigation Architecture for Load Balancing User Demands in Distributed Information Systems", IEICE Trans. Commun., Vol.E84-B, No.10, pp. 1085-1093 (2001)

(14) X. Lu, Y. Zhou M. Tasaka, I. Luque R. Takanuki, Y. Kuba, and K. Mori: "Agent-Based Rating Oriented Information Provision and Reallocation for High-Assurance in Open and Dynamic Environments", Proc. IEEE Conf. on ICDCS2004, pp. 656-661 (2003)

(15) S. Williams and E. W. Fox: "Caching proxies: Limitation and potentials", Proc. 4th Int. World Wide Web Conf. (1995)

Xiaodong Lu (Non-member) received the B.S. and M.S. de-

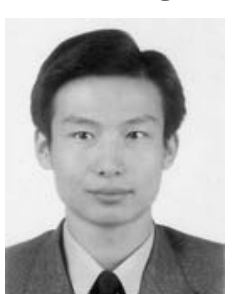
gree in Electronic Engineering and Computer Science from Lanzhou University, P.R.China, in 1997 and 2000, respectively. Presently, he is a doctoral student of the Graduate School of Information Science and Engineering, Tokyo Institute of Technology. His research interests include distributed and high-assurance information systems and mobile agent.
Helene Arfaoui (Non-member) received M.S. degree in

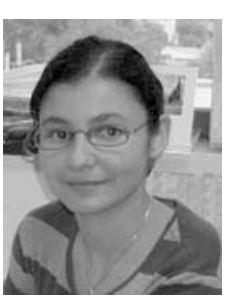
telecommunications technologies from both ENST Bretagne in France and Aston University in England in 1997. In 1998, she joined the Tokyo Institute of Technology, Japan, as a Ph.D. student and received Ph.D. degree in Computer Science in 2002. Her research interests are high-assurance system and autonomy of mobile agents. She is a member of the IEEE society.

Kinji Mori (Member) received the B.S., M.S. and Ph.D.

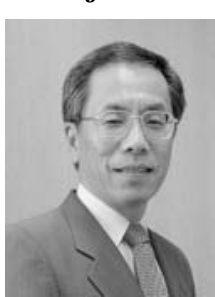
degrees in the Electrical Engineering from Waseda University, Japan in 1969,1971 and 1974, respectively. From 1974 to 1997 he was in System Development Lab., Hitachi, Ltd. In 1997 he joined Tokyo Institute of Technology, Tokyo, Japan as a professor. His research interests include the distributed computing, the fault tolerant computing and the mobile agent. He proposed Autonomous Decentralized Systems (ADS) in 1977 and since then he has been involved in the research and development of ADS. He is a Fellow of IEEE and a member of IEICE, IPSJ and SICE, Japan. 